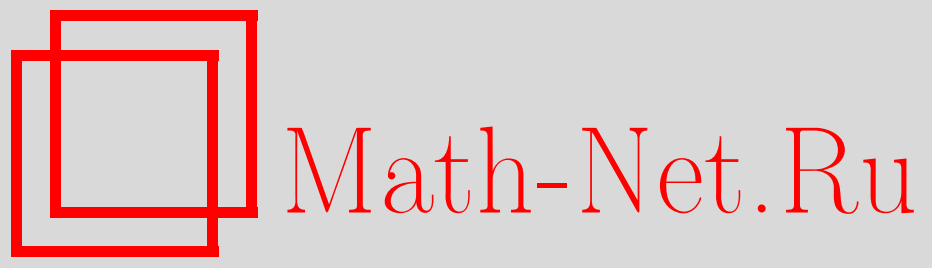

D. Varron, A note on weak convergence, large deviations, and the bounded approximation property, Теория вероятн. и ее примен., 2014, том 59, выпуск 1, 130-149

DOI: https://doi.org/10.4213/tvp4553

Использование Общероссийского математического портала Math-Net.Ru подразумевает, что вы прочитали и согласны с пользовательским соглашением http://www . mathnet.ru/rus/agreement

Параметры загрузки:

IP : 54.196 .121 .252

26 апреля 2023 г., 16:58:54

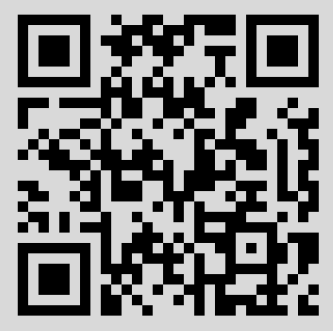




\title{
A NOTE ON WEAK CONVERGENCE, LARGE DEVIATIONS, AND THE BOUNDED APPROXIMATION PROPERTY
}

\begin{abstract}
Для банахова пространства $(E,\|\cdot\|)$ со свойством ограниченной аппроксимации мы предлагаем парадигму, которая может быть использована для установления принципа больших уклонений для последовательности $\left(X_{n}\right)_{n \geqslant 1}$ случайных элементов со значениями в $E$. Похожая парадигма предлагается для установления слабой сходимости $\left(X_{n}\right)_{n} \geqslant 1$ к плотной борелевской мере в $(E,\|\cdot\|)$. Затем мы используем эти инструменты для доказательства функционального предельного закона повторного логарифма для сглаженного эмпирического процесса в гёльдеровой топологии.
\end{abstract}

Ключевые слова и фразы: большие уклонения, слабая сходимость, эмпирические процессы, функциональные предельные законы повторного логарифма.

\section{Introduction and statement of the results.}

1.1. Introduction. The theories of weak convergence and large deviations of a sequence of random elements taking values in a Banach space $(E,\|\cdot\|)$ play an important role in asymptotic statistics. These theories are now very well understood when $E$ is finite dimensional. The scope of this article is to bring a contribution in the investigation on how such computational criteria can be to carried over the infinite dimensional case, by making use of suitable approximations of the identity function of $E$ by finite rank linear operators. This idea is definitely not new, as many research works have explicitly or implicitly involved such approximation methods, for particular Banach spaces. The oldest and most typical example is weak convergence in the space $C([0,1])$ of continuous real functions on $[0,1]$, endowed with the sup norm (see, e.g., [4, p. 55]). In that case, weak convergence of $\left(X_{n}\right)_{n \geqslant 1}$ to $X$ is equivalent to:

(i) Finite dimensional weak convergence: for each integer $p \geqslant 1$ and for each $t_{1}, \ldots, t_{p} \in[0,1]^{p}$, the sequence $\left(X_{n}\left(t_{1}\right), \ldots, X_{n}\left(t_{p}\right)\right)_{n \geqslant 1}$ converges weakly to $\left(X\left(t_{1}\right), \ldots, X\left(t_{p}\right)\right)$;

${ }^{*}$ Laboratoire de Mathématiques de Besançon, UMR CNRS 6623, Université de FrancheComté, Besançon, France; e-mail: davit.varron@univ-fcomte.fr 
(ii) Tightness criterion: for each $\varepsilon>0$ there exists $\eta>0$ such that

$$
\limsup _{n \rightarrow \infty} \mathbf{P}\left(\sup _{\substack{t, t^{\prime} \in[0,1],\left|t^{\prime}-t\right|<\eta}}\left|X_{n}\left(t^{\prime}\right)-X_{n}(t)\right|>\varepsilon\right) \leqslant \varepsilon .
$$

That paradigm was then extended to general spaces of bounded functions (see, e.g., [18, Chap. 1.5]). A very similar paradigm was also established by Arcones [1] for treating large deviation principles in such spaces. The Dawson-Gärtner theorem also provides such a criterion in the setup of spaces that are projective limits of finite dimensional spaces (see, e.g., [7, Chap. 4.6]). The main advantage of such kind of paradigm is that it may allow one to reduce asymptotic properties of a sequence of processes to those of its finite dimensional evaluations, whose properties can be handled more easily (besides Lévy's continuity theorem, see, e.g., [7, Chap. 2], for an overview of techniques in the large deviation setup). Several crucial research works have been made to extend these techniques of finite dimensional approximations to more general frameworks. A nonexhaustive list of references shall follow in that section. The scope of the present article is to show that the majority of these results can be contained in the more general framework of Banach spaces satisfying the bounded approximation property (see subsection 1.2 below). Our results (and their relevance in regard of the existing results in this domain) appear in subsection 1.3.

1.2. The mathematical framework. Before stating our results, we need to give more precisions on the mathematical framework. Given a real function $\Psi$ defined on a set $T$, and given $T_{0} \subset T$ we write

$$
\|\Psi\|_{T_{0}}:=\sup _{t \in T_{0}}|\Psi(t)|
$$

when this quantity is finite. Given a metric space $(F, d)$ and $K \subset F$, we write $B(f, \varepsilon):=\{y \in F, d(y, f)<\varepsilon\}$ and

$$
K^{\varepsilon}:=\bigcup_{f \in K} B(f, \varepsilon) .
$$

Throughout this article, we assume that the Banach space $(E,\|\cdot\|)$ has the so-called bounded approximation property (see, e.g., [13, Definition 1.e.11]). Denote by $\mathscr{L}(E)$ the space of all continuous linear mappings from $(E,\|\cdot\|)$ to $(E,\|\cdot\|)$, and by $\mathscr{O}_{\mathrm{c}}$ the topology on $\mathscr{L}(E)$ of uniform convergence on each compact $K \subset E$. Let $\left(I_{\alpha}\right)_{\alpha \in \Upsilon}$ be a net of finite rank linear operators from $E$ to $E$. The space $(E,\|\cdot\|)$ is said to satisfy the bounded approximation property (BAP for short) with the net $\left(I_{\alpha}\right)_{\alpha \in \Upsilon}$ when

$$
\begin{aligned}
& \lim _{\alpha \in \Upsilon} I_{\alpha}=I_{E} \quad \text { in the space }\left(\mathscr{L}(E), \mathscr{O}_{\mathrm{c}}\right), \\
& \sup _{\alpha}\left\|I_{\alpha}\right\|<\infty .
\end{aligned}
$$


Here $I_{E}$ denotes the identity on $E$, and ||$|L| \|:=\sup \{|L(x)|, x \in E,\|x\|=1\}$ for $L \in \mathscr{L}(E)$.

We point out some typical cases of spaces having the BAP.

1. The spaces having a finite dimensional Schauder decomposition. A Banach space is said to have a finite dimensional Schauder decomposition on a sequence of nonzero finite dimensional subspaces $\left(E_{i}\right)_{i \geqslant 1}$ whenever, for any $e \in E$ there exist a unique pair of sequences $\left(\lambda_{i}(e)\right)_{i \geqslant 1} \in \mathbf{R}^{N}$ and $\left(e_{i}(e)\right)_{i \geqslant 1} \in E_{1} \times E_{2} \times \cdots$ such that

$$
\lim _{n \rightarrow \infty} \sum_{i=1}^{n} \lambda_{i}(e) e_{i}=e .
$$

Now we can set, for $i \geqslant 1$

$$
I_{i}(e):=\sum_{j=1}^{i} \lambda_{j}(e) e_{j},
$$

which defines a sequence of uniformly bounded operators (for more details see, e.g., [16]). Hence any finite dimensional Schauder decomposable space $E$ fulfills the BAP, with $\Upsilon=\mathbf{N}$ and $\left(I_{i}\right)_{i \in \mathbf{N}}$ as in (1.5).

2. The space $\left(L^{\infty}(T),\|\cdot\|_{T}\right)$ of real bounded functions on a set $T$. In that case, $\Upsilon$ is taken as the set of all possible elements of the form $\left(\mathscr{A}, \mathscr{S}_{\mathscr{A}}\right)$, where $\mathscr{A}$ is a finite partition of $T$ and $\mathscr{S}_{\mathscr{A}}=\left\{t_{A}, A \in \mathscr{A}\right\} \subset E$ is finite and satisfies $t_{A} \in A$ for each $A \in \mathscr{A} . \Upsilon$ is endowed with the order $\left(\mathscr{A}, \mathscr{S}_{\mathscr{A}}\right) \prec\left(\mathscr{A}^{\prime}, \mathscr{S}_{\mathscr{A}^{\prime}}\right)$ when $\mathscr{S}_{\mathscr{A}} \subset \mathscr{S}_{\mathscr{A}}$ and $\mathscr{A}^{\prime}$ is nested into $\mathscr{A}$. Then $\left(L^{\infty}(T),\|\cdot\|_{T}\right)$ satisfies

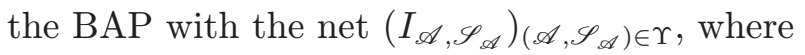

$$
I_{\mathscr{A}, \mathscr{S}_{\mathscr{A}}}(\Psi):=\sum_{A \in \mathscr{A}} \Psi\left(t_{A}\right) 1_{A}, \quad \Psi \in L^{\infty}(T) .
$$

Denote by $\mathscr{B}(E)$ the Borel $\sigma$-algebra of $(E,\|\cdot\|)$. From now on, we consider a probability space $(\Omega, \mathscr{A}, \mathbf{P})$ and a sequence $\left(X_{n}\right)_{n \geqslant 1}$ of mappings from $\Omega$ to $E$, for which $I_{\alpha}\left(X_{n}\right)$ is Borel measurable for each $n \geqslant 1, \alpha \in \Upsilon$. We call random element each mapping having that measurability property. There are situations in which $X_{n}$ is Borel measurable for no $n \geqslant 1$. One example where this lack of measurability occurs is when $E=L^{\infty}([0,1])$ and $X_{n}$ is the empirical distribution function induced by a uniform sample $\left(U_{1}, \ldots, U_{n}\right)$. One way to tackle that problem is to introduce inner and outer probabilities, namely,

$$
\begin{aligned}
& \mathbf{P}^{*}(A):=\inf \{\mathbf{P}(B), B \in \mathscr{A}, A \subset B\}, \quad A \subset \Omega, \\
& \mathbf{P}_{*}(A):=\sup \{\mathbf{P}(B), B \in \mathscr{A}, B \subset A\}, \quad A \subset \Omega .
\end{aligned}
$$

That framework has first been introduced by Hoffmann-Jørgensen [12] to define weak convergence of (nonnecessarily Borel) random elements to a tight Borel probability measure on $E$. Arcones [1] also adopted that framework to investigate large deviations in the particular space $\left(L^{\infty}(T),\|\cdot\|_{T}\right)$. 


\subsection{Statement of the results.}

1.3.1. Weak convergence. We first recall the notion of asymptotic tightness (see, e.g., [18, Definition 1.3.7, p. 21]).

$\mathrm{D}$ e f i n it i o n 1.1. A sequence $X_{n}$ of random elements is said to be asymptotically tight when, for each $\varepsilon>0$, there exists a compact set $K$ for which

$$
\limsup _{n \rightarrow \infty} \mathbf{P}^{*}\left(X_{n} \notin K^{\delta}\right) \leqslant \varepsilon, \quad \text { for every } \delta>0 .
$$

Our first result has a straightforward proof. We state it here because we could not find such a result in the existing literature, to the best of our knowledge.

Theorem 1.1. Let $\left(X_{n}\right)_{n \geqslant 1}$ be a sequence of random elements taking values in a Banach space $(E,\|\cdot\|)$ which satisfies the BAP for a net $\left(I_{\alpha}\right)_{\alpha \in \Upsilon}$. The sequence $\left(X_{n}\right)_{n \geqslant 1}$ is asymptotically tight if and only if ity;

(A1) For each $\alpha \in \Upsilon$, the sequence $\left(I_{\alpha}\left(X_{n}\right)\right)_{n \geqslant 1}$ is bounded in probabil-

(A2) for each $\varepsilon>0$ we have

$$
\lim _{\alpha \in \Upsilon} \limsup _{n \rightarrow \infty} \mathbf{P}^{*}\left(\left\|I_{\alpha}\left(X_{n}\right)-X_{n}\right\| \geqslant \varepsilon\right) \leqslant \varepsilon .
$$

As a consequence, if $X$ denotes a Borel random variable taking values in $E$, then $X_{n} \rightarrow \mathscr{L} X$ if and only if (A2) holds together with the following assertion:

( $\left.\mathrm{A}^{\prime} 1\right)$ For each $\alpha \in \Upsilon$ we have

$$
I_{\alpha}\left(X_{n}\right) \rightarrow \mathscr{L} I_{\alpha}(X)
$$

1.3.2. Large deviations. Before stating our result in large deviation theory, we need to introduce some definitions. We call a rate function on $(E,\|\cdot\|)$ any mapping $J$ from $E$ to the extended semiline $[0, \infty]$, such that the set $\{x \in E, J(x) \leqslant a\}$ is compact for each finite $a \geqslant 0$. Given $F \subset E$ we write $J(F):=\inf \{J(x), x \in F\}$.

$\mathrm{D}$ e f i n i t i o n 1.2 (see [2]). Let $v_{n} \uparrow \infty$ be a sequence of positive constants and let $J$ be a rate function $(E,\|\cdot\|)$. A sequence of random elements $\left(X_{n}\right)_{n \geqslant 1}$ is said to satisfy the large deviation principle (LDP for short) for $v_{n}$ and the rate function $J$ when the two following conditions hold:

For each closed set $F \subset E: \quad \limsup _{n \rightarrow \infty} \frac{1}{v_{n}} \ln \mathbf{P}^{*}\left(X_{n} \in F\right) \leqslant-J(F)$;

For each open set $O \subset E$ : $\quad \liminf _{n \rightarrow \infty} \frac{1}{v_{n}} \ln \mathbf{P}_{*}\left(X_{n} \in O\right) \geqslant-J(O)$. 
Whenever no ambiguity is possible we shall avoid mentioning $v_{n}$.

Large deviations principles have been subject of many investigations since the seminal works of Cramér [6] and Chernoff [5]. We refer to the book of Dembo and Zeitouni [7] for a strong theoretical overview of that topic. We shall now state our second result, then discuss its relevance with respect to the existing literature.

Theorem 1.2. Let $v_{n}$ be a sequence of positive constants fulfilling $v_{n} \uparrow \infty$. Let $\left(X_{n}\right)_{n \geqslant 1}$ be a sequence of random elements in E. Consider the following conditions:

(B1) For each $\alpha \in \Upsilon$, the sequence $\left(I_{\alpha}\left(X_{n}\right)\right)_{n \geqslant 1}$ satisfies the LDP in $(E,\|\cdot\|)$ for $v_{n}$ and the rate function $J_{\alpha}$;

(B2) for each $\varepsilon>0$ we have

$$
\lim _{\alpha \in \Upsilon} \limsup _{n \rightarrow \infty} \frac{1}{v_{n}} \ln \mathbf{P}^{*}\left(\left\|X_{n}-I_{\alpha}\left(X_{n}\right)\right\|>\varepsilon\right) \leqslant-\frac{1}{\varepsilon}
$$

(b1) the sequence $\left(X_{n}\right)_{n \geqslant 1}$ satisfies the LDP for the rate function $J$. Then (B1), (B2) together imply (b1), with $J$ defined as

$$
J(x):=\sup _{\alpha \in \Upsilon} J_{\alpha}\left(I_{\alpha}(x)\right), \quad x \in E .
$$

Moreover, (b1) implies both (B1) and (B2) with

$$
J_{\alpha}(x):=\inf \left\{J(y), I_{\alpha}(y)=x\right\} .
$$

Discussion. 1. A characterization of large deviations in a Polish space has been established by Lynch and Sethuraman [14], where they introduce the notion of weak LDP and of tightness (in the large deviations sense). Varron [19] investigated how their works could be translated to the context of separable, Schauder decomposable spaces, and derived a necessary and sufficient criterion for which there is a very natural interpretation in several spaces (see section 2 in the present paper for more details). Arcones [1], [2] investigated the case of the nonseparable spaces $L^{\infty}(T)$ and also obtained a necessary and sufficient criterion (finite dimensional LDP coupled with an equicontinuity condition) which is again easily interpretable (see Theorem 3.1 in the sequel). The framework of spaces having the bounded approximation property is of course wider. However, a close look at the proof of Theorem 1.2 shows that the involved arguments deeply root in the result of Arcones, as we utilize the identification a Banach space $E$ to a suitable subspace of $L^{\infty}\left(E^{*}\right)$, where $E^{*}$ is the topological dual of $(E,\|\cdot\|)$. Also note that Pukhalskii [15, Theorem 2] had previously established a very similar result in the Skorokhod space on $\mathbf{R}^{d}$.

2. Many sufficient (and sometimes necessary) criteria have been established to prove a LDP in a wide topological framework (Hausdorff regular 
spaces, metric spaces). Important examples of these criteria are Bryc's inverse Varadhan lemma (see, e.g., [7, Chap. 4.4]), the abstract version of the Gärtner-Ellis theorem (see, e.g., [7, Theorem 4.5.20, p. 157]), or Pukhalskii's criterion (see [15, Theorem 1]). The main relevance of Theorem 1.2 is that, depending on the problem, one can choose the net $\left(I_{\alpha}\right)_{\alpha \in \Upsilon}$ so that:

(a) The $\left(I_{\alpha}\right)_{\alpha \in \Upsilon}$ are linear continuous, which defines a quite restricted subclass of continuous applications on $E$. That remark has to be taken into account when comparing Theorem 1.2 to Bryc's inverse Varadhan lemma and its refinements.

(b) The $\left(I_{\alpha}\right)_{\alpha \in \Upsilon}$ are of finite rank but are not necessarily related to continuous linear forms on $E$. This may play an important role when $E^{*}$ has a representation uneasy to handle (which is the case of most of the spaces appearing in the present article). That remark is important when comparing Theorem 1.2 with the abstract version of the Gärtner-Ellis theorem.

(c) Also note that the Dawson-Gärtner theorem (see, e.g., [7, section 4.6, Theorem 4.6.1]) provides a related result in the framework of spaces that are projective limits of finite dimensional spaces. The later framework has many conceptual similarities with the present one. However, Theorem 1.2 allows us to handle topologies that are stronger than the natural topology of projective limits.

2. Some applications of Theorem 1.2. Suquet [17] highlighted that his result on weak convergence in separable Schauder decomposable spaces leads to many useful paradigms in usual functional spaces. In this section, we provide a series of results in the same vein, but in the large deviations setup. The first two results hold in the context of Schauder decomposable spaces. They are direct consequences of Theorem 1 in [19], hence the full generality of Theorem 1.2 is not needed for them. The third result is an application to smoothed versions of the uniform empirical process, for which large deviation properties in Hölderian spaces are investigated. Finally, to provide an application of Theorem 1.2 beyond the preceding framework, we establish a fourth result, taking place in $L_{1}(T, \chi, \mu)$, where $(T, \chi, \mu)$ is a measured space, with non- $\sigma$-finite, measure $\mu$. To avoid a lengthy introduction of notation we make use of the notations appearing in [17]. Throughout that section, $v_{n}$ denote a nonrandom positive sequence tending to $\infty$.

2.1. Hölderian spaces. For $0<\alpha<1$, let $H_{\alpha}$ be the space of $\alpha$ Hölder real functions on $[0,1]$ endowed with the norm

$$
\|f\|_{\alpha}=\mathscr{D}(f, \alpha, 1)+\|f\|_{[0,1]},
$$

where

$$
\mathscr{D}(f, \alpha, \delta):=\sup _{\substack{s, t \in[0,1] \\|s-t| \leqslant \delta}} \frac{|f(s)-f(t)|}{|s-t|^{\alpha}} \text { and }\|f\|_{[0,1]}=\sup _{t \in[0,1]}|f(t)| .
$$


Also define the following subspace of $\left(H_{\alpha},\|\cdot\|_{\alpha}\right)$ :

$$
H_{\alpha, 0}:=\left\{f \in H_{\alpha}, \lim _{\delta \rightarrow 0} \mathscr{D}(f, \alpha, \delta)=0\right\} .
$$

Denote by Dy $[0,1]$ the set of dyadic real numbers of $[0,1]$ (a real number is said to be dyadic when it can be written in the form $p \cdot 2^{-k}$, for some integers $p$ and $k$ ). The Schauder decomposition of that space on dyadic blocks of the Faber-Schauder basis provides a nice derivation of Theorem 1.2.

Corollary 2.1. Let $\left(X_{n}\right)_{n \geqslant 1}$ be a sequence of $H_{\alpha, 0}$-valued random elements. Then $\left(X_{n}\right)_{n \geqslant 1}$ satisfies the LDP for some rate function $J$ if and only if

(i) For each $p \geqslant 1$ and $\left(t_{1}, \ldots, t_{p}\right) \in \mathrm{Dy}[0,1]^{p}$, the sequence $\left(X_{n}\left(t_{1}\right), \ldots, X_{n}\left(t_{p}\right)\right)_{n \geqslant 1}$ satisfies the LDP for $v_{n}$ and a rate function $J_{t_{1}, \ldots, t_{p}}$;

(ii) for each $\varepsilon>0$ we have

$$
\lim _{\delta \rightarrow 0} \limsup _{n \rightarrow \infty} \frac{1}{v_{n}} \ln \mathbf{P}\left(\mathscr{D}\left(X_{n}, \alpha, \delta\right)>\varepsilon\right) \leqslant-\frac{1}{\varepsilon} .
$$

Moreover, if (i) and (ii) hold, then $J$ is defined by

$$
J(f):=\sup \left\{J_{t_{1}, \ldots, t_{p}}\left(f\left(t_{1}\right), \ldots, f\left(t_{p}\right)\right), p \geqslant 1,\left(t_{1}, \ldots, t_{p}\right) \in \operatorname{Dy}[0,1]^{p}\right\} .
$$

P r o o $f$. The main argument of the proof is that $\mathscr{D}\left(f, \alpha, 2^{-k}\right)$ is related to $\left\|f-I_{k}(f)\right\|_{\alpha}$, where $I_{k}(f)$ denotes the projection of $f$ on the space spanned by the $k$ first dyadic blocks of the Faber-Schauder basis (see, e.g., [17]). We omit details.

2.2. Sequence spaces and Besov spaces. Theorem 1.2 also implies the following paradigm in sequence spaces $\ell^{p}\left(\mathbf{Z}^{k}\right), 0<p<\infty, k \geqslant 1$. Indeed, every such space admits the natural basis as an unconditional Schauder basis. This leads to the following result, whose proof is omitted.

Corollary 2.2. Let $\left(X_{n}\right)_{n \geqslant 1}$ be a sequence of random elements taking values in $\ell^{p}\left(\mathbf{Z}^{k}\right)$ for $k \geqslant 1, p \in(0, \infty)$. Then $\left(X_{n}\right)_{n \geqslant 1}$ satisfies the LDP for $v_{n}$ and a rate function $J$ if and only if

(i) For each $p \geqslant 1$ and $\left(z_{1}, \ldots, z_{p}\right) \in \mathbf{Z}^{k^{p}}$, the sequence $\left(X_{n}\left(z_{1}\right), \ldots\right.$, $\left.X_{n}\left(z_{p}\right)\right)_{n \geqslant 1}$ satisfies the LDP for a rate function $J_{z_{1}, \ldots, z_{p}}$;

(ii) for each $\varepsilon>0$ we have

$$
\lim _{r \rightarrow \infty} \limsup _{n \rightarrow \infty} \frac{1}{v_{n}} \ln \mathbf{P}\left(\sum_{z \notin\{-r, \ldots, r\}^{k}}\left|X_{n}(z)\right|^{p}>\varepsilon\right) \leqslant-\frac{1}{\varepsilon} .
$$

Any Besov space $B_{p}^{s, q}$ can be identified to a sequence space through the following scheme: taking any multiresolution analysis with regularity $r>s$, 
any $f \in B_{p}^{s, q}$ has the associated decomposition with coefficients $\left(\alpha_{0, k}(f)\right)_{k \in \mathbf{Z}}$,

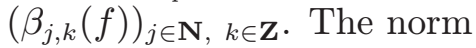

$$
K_{p}^{s, q}(f):=\left\|\alpha_{0, \cdot}\right\|_{\ell^{p}(\mathbf{Z})}+\left(\sum_{j \geqslant 0}\left(2^{j(s+1 / 2-1 / p)}\left\|\beta_{j, \cdot}\right\|_{\ell^{p}(\mathbf{Z})}\right)^{q}\right)^{1 / q}
$$

is equivalent to the natural norm of $B_{p}^{s, q}$. For more details, see [3, Theorem 6.2.5]. Such an identification with sequence spaces leads to the following straightforward result.

Corollary 2.3. Let $\left(X_{n}\right)_{n \geqslant 1}$ be a sequence of random elements taking values in $B_{p}^{s, q}$. Then $\left(X_{n}\right)_{n \geqslant 1}$ satisfies the $L D P$ for $v_{n}$ and a rate function $J$ if and only if

(i) For each $j \geqslant 0$, the sequence $\left(\alpha_{0, .}\left(X_{n}\right), \beta_{0, .}\left(X_{n}\right), \ldots, \beta_{j, .}\left(X_{n}\right)\right)_{n \geqslant 1}$ satisfies the LDP for a rate function $J_{j}$;

(ii) for each $\varepsilon>0$ we have

$$
\lim _{i \rightarrow \infty} \limsup _{n \rightarrow \infty} \frac{1}{v_{n}} \ln \mathbf{P}\left(\sum_{j \geqslant i}\left(2^{j(s+1 / 2-1 / p)}\left\|\beta_{j, \cdot}\right\|_{\ell^{p}(\mathbf{Z})}\right)^{q}>\varepsilon\right) \leqslant-\frac{1}{\varepsilon} .
$$

2.3. The Hölderian law of the iterated logarithm of the smoothed uniform empirical process. In this subsection we establish a large deviation principle for the smoothed uniform empirical process, which is defined as follows: given an i.i.d. sequence $\left(U_{i}\right)_{i \geqslant 1}$ uniformly distributed on $[0,1]$ and given a density probability function $K$, we write

$$
\alpha_{n}(t):=\sqrt{n}\left(\frac{1}{n} \sum_{i=1}^{n} 1_{[0, t]}\left(U_{i}\right)-t\right), \quad \alpha_{n, K, h_{n}}=\alpha_{n} * K_{h_{n}},
$$

where $K_{h}(u)=h^{-1} K\left(h^{-1} u\right), u \in \mathbf{R}, *$ is the convolution operator and $\left(h_{n}\right)_{n \geqslant 1}$ is a sequence of positive constants converging to 0 . Under mild conditions on $K$ and $h_{n}$, Hamadouche [11] has established the weak invariance principle of the sequence of processes $\left(\alpha_{n, K, h_{n}}(\cdot)-\alpha_{n, K, h_{n}}(0)\right)_{n \geqslant 1}$, in the Banach subspace $E=\left\{f \in H_{0, \alpha}, f(0)=0\right\}$. To gain in generality, we work directly on $\alpha_{n, K, h_{n}}$ in the Banach space $H_{0, \alpha}$.

Theorem 2.1. Assume that $n h_{n}^{4} / \log _{2} n \rightarrow \infty$ and $K$ is Lipschitz. Then for any $\alpha \in[0,1 / 2)$ the sequence $\left(2 \log _{2} n\right)^{-1 / 2} \alpha_{n, K, h_{n}}$ satisfies the $L D P$ in $H_{\alpha, 0}$, for the sequence $v_{n}=\log _{2} n$ and the rate function

$$
J(f):=\inf \left\{\int_{0}^{1} g^{2} d \lambda, d f=g . d \lambda\right\}
$$

with the usual convention inf $\varnothing=\infty$.

A consequence of that large deviation result is the functional law of the iterated logarithm for $\alpha_{n, K, h_{n}}$, which is stated here without proof, as the latter involves only standard arguments (see, e.g., [10]). 
Corollary 2.4. Assume that $n h_{n}^{4} / \log _{2} n \rightarrow \infty$ and $K$ is Lipschitz. Then for any $\alpha \in[0,1 / 2)$ the sequence $\left(2 \log _{2} n\right)^{-1 / 2} \alpha_{n, K, h_{n}}$ almost surely has his cluster set in $H_{0, \alpha}$ equal to the Strassen set $\mathscr{S}:=\left\{f \in H_{0, \alpha}, J(f) \leqslant 1\right\}$.

2.4. Nonseparable $L_{1}$ spaces. Given a measured space $(T, \chi, \mu)$, with $\mu$ non- $\sigma$-finite, a natural directed net of approximating finite rank op-

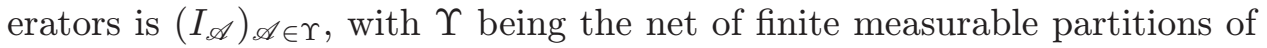
$T$ and

$$
I_{\mathscr{A}}: f \rightarrow \sum_{A \in \mathscr{A}} \mu(A)^{-1} \int_{A} f d \mu 1_{A}
$$

with the convention that $1 /(+\infty)=0$. An application of Theorem 1.2 in this context leads to the following corollary.

Corollary 2.5. Let $\left(X_{n}\right)_{n \geqslant 1}$ be a sequence of random elements taking values in $L_{1}(T, \chi, \mu)$ for which $\int_{A} X_{n} d \mu$ is Borel measurable for any $A \in \chi$ and $n \geqslant 1$. Then $\left(X_{n}\right)_{n \geqslant 1}$ satisfies the LDP for $v_{n}$ and a rate function $J$ if and only if

(i) For each finite measurable partition $A_{1}, \ldots, A_{p}$, the sequence

$$
\left(\int_{A_{1}} X_{n} d \mu, \ldots, \int_{A_{p}} X_{n} d \mu\right)
$$

satisfies the LDP for a rate function $J_{A_{1}, \ldots, A_{p}}$;

(ii) for each $\varepsilon>0$ and $M>0$, there exists $A_{1}, \ldots, A_{p}$ such that

$$
\limsup _{n \rightarrow \infty} \frac{1}{v_{n}} \ln \mathbf{P}\left(\sum_{j=1}^{p} \int_{A_{j}}\left|X_{n}-\mu\left(A_{j}\right)^{-1} \int_{A_{j}} X_{n} d \mu\right| d \mu>\varepsilon\right) \leqslant-M .
$$

\section{Proofs.}

3.1. Proof of Theorem 1.1. From now on, we shall say that a sequence $\left(\alpha_{n}\right)$ belonging to the ordered set $(\Upsilon, \prec)$ is $\prec$-increasing when the terms of $\left(\alpha_{n}\right)$ satisfy $\alpha_{n} \prec \alpha_{n+1}$ for all $n$.

We shall first prove that (A1) and (A2) are necessary. Proving (A1) is straightforward, as $I_{\alpha}\left(K^{\delta}\right)$ is bounded for any compact set $K$ and any $\delta>0, \alpha \in \Upsilon$. To prove (A2), fix $\varepsilon>0$ and choose $K$ to fulfill (1.6). By the bounded approximation property, there exists $\alpha_{0}$ satisfying, for each $\alpha \succeq \alpha_{0}$,

$$
\sup _{e \in K}\left\|I_{\alpha}(e)-e\right\| \leqslant \varepsilon,
$$

from where

$$
\sup _{e \in K^{\varepsilon}}\left\|I_{\alpha}(e)-e\right\| \leqslant\left(1+\sup _{\alpha \in \Upsilon}\left\|I_{\alpha}\right\| \|\right) \varepsilon .
$$

The conclusion follows from

$$
\left\{\left\|I_{\alpha}\left(X_{n}\right)-X_{n}\right\| \geqslant\left(1+\sup _{\alpha \in \Upsilon}\left\|I_{\alpha}\right\| \|\right) \varepsilon\right\} \subset\left\{X_{n} \notin K^{\varepsilon}\right\}
$$


Assume that (A1) and (A2) hold. To show that $\left(X_{n}\right)_{n \geqslant 1}$ is pretight, fix $\varepsilon>0$ arbitrarily and set $\varepsilon_{k}=2^{-k} \varepsilon, k \geqslant 1$. Further consider a strictly increasing, integer-valued sequence $\left(n_{k}\right)_{k \geqslant 1}$ as well as a $\prec$-increasing sequence $\left(\alpha_{k}\right)_{k \geqslant 1}$ and a real-valued sequence $\left(M_{k}\right)_{k \geqslant 1}$ satisfying

$$
\sup _{n_{k} \leqslant n} \mathbf{P}^{*}\left(\left\{\left\|X_{n}-I_{\alpha_{k}}\left(X_{n}\right)\right\|>\varepsilon_{k}\right\} \cup\left\{\left\|I_{\alpha_{k}}\left(X_{n}\right)\right\| \geqslant M_{k}\right\}\right) \leqslant \varepsilon_{k} .
$$

Such sequences can be built by applying (A2) to build $\left(\alpha_{k}\right)_{k \geqslant 1}$, then (A1) to build $\left(M_{k}\right)_{k \geqslant 1}$ and $\left(n_{k}\right)_{k \geqslant 1}$. Now define $A_{k}:=\left\{e \in E\right.$ : $\left\|e-I_{\alpha_{k}}(e)\right\| \leqslant \varepsilon_{k}$ and $\left.\left\|I_{\alpha_{k}}(e)\right\|<M_{k}\right\}$. For each $k \geqslant 1$ we can write

$$
A_{k} \subset \bigcup_{i=1}^{p_{k}} B\left(x_{i, k}, 2 \varepsilon_{k}\right)
$$

for a finite collection $x_{1, k}, \ldots, x_{p_{k}, k}$. Here $B(x, \varepsilon)$ stands for the open ball with centre $x$ and radius $\varepsilon$. Now set

$$
B_{k}=\bigcup_{i=1}^{p_{k}} \overline{B\left(x_{i, k}, 2 \varepsilon_{k}\right)}
$$

Obviously $K:=\bigcap B_{k}$ is compact. Moreover, we have, for each $k \geqslant 1$,

$$
\liminf _{n \rightarrow \infty} \mathbf{P}_{*}\left(X_{n} \in \bigcap_{i=1}^{k} B_{i}\right) \geqslant \liminf _{n \rightarrow \infty} \mathbf{P}_{*}\left(X_{n} \in \bigcap_{i=1}^{k} A_{i}\right) \geqslant 1-\sum_{i=1}^{k} \varepsilon_{i} \geqslant 1-\varepsilon
$$

From here the proof of asymptotic tightness is completed as in [18, p. 36-37]. To complete the proof of Theorem 1.1, notice that the class $\mathscr{F}$ of functions $f: E \rightarrow \mathbf{R}$ of the form

$$
f:=g \circ I_{\alpha}, \quad \alpha \in \Upsilon, \quad g \text { is bounded and continuous on } I_{\alpha}(E),
$$

is an algebra and a vector lattice that contains the constant functions and separates the points of $E$. Since $f\left(X_{n}\right)$ is Borel measurable for each $f \in \mathscr{F}$ and $\left(X_{n}\right)_{n \geqslant 1}$ is pretight, we can combine Theorem 1.3.9 with Lemmas 1.3.12 and 1.3 .13 in $\left[18\right.$, p. 25] to conclude that $X_{n} \rightarrow \mathscr{L} X$.

3.2. Proof of Theorem 1.2. Denote by $E^{\prime}$ the dual of $(E,\|\cdot\|)$ and by $B\left(E^{\prime}\right)$ its unit ball. A consequence of the work of Arcones on large deviations in $L^{\infty}(T)$ is the following paradigm in $(E,\|\cdot\|$ ) (it is a slight adaptation of Corollary 3.6 in [1]).

Theorem 3.1 (see [2]). Let $\left(X_{n}\right)_{n \geqslant 1}$ be a sequence of random elements taking values in a Banach space $E$. Consider the following assertions.

(D1) For each $p \geqslant 1$ and $\left(L_{1}, \ldots, L_{p}\right) \in E^{\prime p}$ the sequence $\left(L_{1}\left(X_{n}\right), \ldots, L_{p}\left(X_{n}\right)\right)_{n \geqslant 1}$ satisfies the LDP for $v_{n}$ and the rate function $J_{L_{1}, \ldots, L_{p}}$ on $\mathbf{R}^{p}$. 
(D2) There exists $\lambda>0$ such that, for each $\varepsilon>0$, there exists a finite partition $\mathscr{L}$ of $\lambda B\left(E^{\prime}\right)$ satisfying

$$
\limsup _{n \rightarrow \infty} \frac{1}{v_{n}} \ln \mathbf{P}^{*}\left(\max _{B \in \mathscr{L}} \sup _{L, L^{\prime} \in B}\left|L\left(X_{n}\right)-L^{\prime}\left(X_{n}\right)\right|>\varepsilon\right) \leqslant-\frac{1}{\varepsilon} .
$$

(d1) $\left(X_{n}\right)_{n \geqslant 1}$ satisfies the LDP in $(E,\|\cdot\|)$ for $v_{n}$ and the rate function $J$. Then (D1) and (D2) together imply (d1) with $J$ defined as

$$
J(e):=\sup \left\{J_{L_{1}, \ldots, L_{p}}\left(L_{1}(e), \ldots, L_{p}(e)\right), p \geqslant 1,\left(L_{1}, \ldots, L_{p}\right) \in E^{\prime p}\right\},
$$

where $e \in E$.. Moreover if (d1) holds, then

1) $B\left(E^{\prime}\right)$ is totally bounded with respect to the pseudo distance

$$
\rho_{0}:=\sum_{k=1}^{\infty} 2^{-k}\left(\rho_{k} \wedge 1\right)
$$

with

$$
\rho_{k}\left(L, L^{\prime}\right):=\sup \left\{\left|L(e)-L^{\prime}(e)\right|, \quad J(e) \leqslant k\right\}, \quad\left(L, L^{\prime}\right) \in{E^{\prime}}^{2}, \quad k \geqslant 1 ;
$$

2) (D1) holds with

$$
J_{L_{1}, \ldots, L_{p}}\left(l_{1}, \ldots, l_{p}\right):=\inf \left\{J(e), L_{i}(e)=l_{i} \text { for all } i=1, \ldots, p\right\} ;
$$

2) (D2) holds; in addition we have, for each $\lambda>0, \varepsilon>0$,

$$
\lim _{\delta \rightarrow 0} \limsup _{n \rightarrow \infty} \frac{1}{v_{n}} \ln \mathbf{P}^{*}\left(\sup _{\substack{L, L^{\prime} \in \lambda B\left(E^{\prime}\right) \\ \rho_{0}\left(L, L^{\prime}\right)<\delta}}\left|L\left(X_{n}\right)-L^{\prime}\left(X_{n}\right)\right|>\varepsilon\right) \leqslant-\frac{1}{\varepsilon} .
$$

3.2.1. Proof that (B1), (B2) imply (b1). Assume that (B1) and (B2) hold. To prove (b1) we shall make use of Theorem 3.1.

We shall first prove that (D1) holds. Consider a finite collection $\left(L_{1}, \ldots, L_{p}\right) \in B\left(E^{\prime}\right)^{p}$, and define the finite rank application $\bar{L}: E \rightarrow \mathbf{R}^{p}$ by $\bar{L}(x)=\left(L_{1}(x), \ldots, L_{p}(x)\right)$. We shall endow $\mathbf{R}^{p}$ with the usual max norm

$$
|\bar{l}|_{p}:=\max _{i=1, \ldots, p}\left|\bar{l}_{i}\right| .
$$

First note that, for any $\alpha \in \Upsilon$, the sequence $\left(\bar{L} \circ I_{\alpha}\left(X_{n}\right)\right)_{n \geqslant 1}$ satisfies the LDP for the following rate function:

$$
J_{\alpha, \bar{L}}(\bar{l}):=\inf \left\{J_{\alpha}(e), \bar{L}(e)=\bar{l}\right\}, \quad \bar{l} \in \mathbf{R}^{p},
$$

by (B1) together with the contraction principle. Proving (D1) is equivalent to proving that $\left(\bar{L}\left(X_{n}\right)\right)_{n \geqslant 1}$ satisfies the LDP in $\mathbf{R}^{p}$ for $v_{n}$ and for some rate function. We shall prove it through the following lemma. 
Lemma 3.1. Under the assumptions of Theorem 1.2, the sequence $\left(\bar{L}\left(X_{n}\right)\right)$ satisfies the LDP for $v_{n}$ and for the following rate function:

$$
J_{\bar{L}}(\bar{l}):=\sup \left\{J_{\alpha, \bar{L}}(\bar{l}), \alpha \in \Upsilon\right\} .
$$

Moreover, we have the equality

$$
J_{\alpha}=\sup \left\{J_{\alpha, \bar{L}}, p \geqslant 1, L_{1}, \ldots, L_{p} \in \mathrm{B}\left(\mathrm{E}^{\prime}\right)^{p}\right\} .
$$

P r o o f. First note that the second part of Lemma 3.1 (equality (3.10)) is a consequence of (B1) together with the contraction principle. Indeed, we know that $\left(I_{\alpha}\left(X_{n}\right)\right)_{n \geqslant 1}$ satisfies the LDP in $(E,\|\cdot\|)$ for $v_{n}$ and for a rate function and hence it satisfies (D2). Now since, for each $\bar{L}$, the sequence $\left(\bar{L}\left(I_{\alpha}\left(X_{n}\right)\right)\right)_{n \geqslant 1}$ satisfies the LDP for $v_{n}$ and for $J_{\alpha, \bar{L}}$, we can apply part 1 of Theorem 3.1. From that, we conclude that $\left(X_{n}\right)_{n \geqslant 1}$ satisfies the LDP for the rate function $J_{\alpha}$, which implies equality (3.10) by uniqueness of the rate function.

The proof of the first part of Lemma 3.1 is naturally divided into two steps.

Step 1. We shall first prove the open sets part of Lemma 3.1. Consider an open set $O \subset \bar{L}(E)$ and fix $\delta>0$. Assuming that $J_{\bar{L}}(O)<\infty$ (non trivial case), we can choose $\bar{l} \in O$ fulfilling $J_{\bar{L}}(O) \leqslant J_{\bar{L}}(\bar{l}) \leqslant J_{\bar{L}}(O)+\delta$, as well as $\varepsilon_{0}>0$ fulfilling $\left|\bar{l}^{\prime}-\bar{l}\right|_{p}<2 \varepsilon_{0} \Rightarrow \bar{l}^{\prime} \in O$. Now write, for any $\alpha \in \Upsilon$ and $0<\varepsilon<\varepsilon_{0}$ :

$$
\begin{aligned}
\mathbf{P}_{*}\left(\bar{L}\left(X_{n}\right) \in O\right) \geqslant & \mathbf{P}_{*}\left(\left|\bar{L}\left(X_{n}\right)-\bar{l}\right|_{p}<2 \varepsilon_{0}\right) \\
\geqslant & \mathbf{P}_{*}\left(\left|\bar{L}\left(I_{\alpha}\left(X_{n}\right)\right)-\bar{l}\right|_{p}<\varepsilon_{0}\right) \\
& -\mathbf{P}^{*}\left(\left\|I_{\alpha}\left(X_{n}\right)-X_{n}\right\|>\varepsilon\right) .
\end{aligned}
$$

Note that (3.11) is a consequence of the fact that $\bar{L}$ has norm equal to 1 . Now choose $\varepsilon>0$ so as $1 / \varepsilon>J_{\bar{L}}(O)+2 \delta$, and $\alpha \in \Upsilon$ so as

$$
\limsup _{n \rightarrow \infty} \frac{1}{v_{n}} \ln \mathbf{P}^{*}\left(\left\|X_{n}-I_{\alpha}\left(X_{n}\right)\right\|>\varepsilon\right) \leqslant-\frac{1}{\varepsilon} .
$$

We obtain

$$
\liminf _{n \rightarrow \infty} \frac{1}{v_{n}} \ln \mathbf{P}_{*}\left(\bar{L}\left(X_{n}\right) \in O\right) \geqslant-J_{\alpha, \bar{L}}(O)-2 \delta \geqslant-J_{\bar{L}}(O)-2 \delta,
$$

which concludes the proof of the open sets part of Lemma 3.1.

As $\bar{L}(E)$ is finite dimensional (hence Polish), we shall deal with the closed sets part of Lemma 3.1 by making use of the results of Lynch and Sethuraman [14]. Note however that, as inner and outer probabilities are involved in our context, a slight adaptation of their results has to be used. 
We shall first show that $\left(\bar{L}\left(X_{n}\right)\right)_{n \geqslant 1}$ is LD-tight in the sense of [14, Definition 2.4], which means that for every $\varepsilon>0$, there exists $A>0$ fulfilling

$$
\limsup _{n \rightarrow \infty} \frac{1}{v_{n}} \ln \mathbf{P}^{*}\left(\max _{i=1, \ldots, p}\left|L_{i}\left(X_{n}\right)\right| \geqslant A\right) \leqslant-\frac{1}{\varepsilon} .
$$

To prove this, fix $\varepsilon>0$ and choose $\alpha_{0}$ fulfilling, for all large $n$,

$$
\mathbf{P}^{*}\left(\left\|I_{\alpha_{0}}\left(X_{n}\right)-X_{n}\right\|>1\right) \leqslant \exp \left(-\frac{v_{n}}{\varepsilon}\right) .
$$

Now, as $\left\|I_{\alpha_{0}}\left(X_{n}\right)\right\|$ satisfies the LDP in $\mathbf{R}$, it is tight, and we can find $A_{0}>0$ fulfilling, for all large $n$,

$$
\mathbf{P}^{*}\left(\left\|I_{\alpha_{0}}\left(X_{n}\right)\right\| \geqslant A_{0}\right) \leqslant \exp \left(-\frac{v_{n}}{\varepsilon}\right) .
$$

As each $L_{i}, i=1, \ldots, p$, has a norm equal to one, (3.12) follows by choosing $A=A_{0}+1$ and by making use of the triangle inequality.

It remains to show that, for any compact $K \subset \bar{L}(E)$ we have

$$
\limsup _{n \rightarrow \infty} \frac{1}{v_{n}} \ln \mathbf{P}^{*}\left(\bar{L}\left(X_{n}\right) \in K\right) \leqslant-J_{\bar{L}}(K) .
$$

To prove this, consider a compact set $K \subset \bar{L}(E)$. Fix $\delta>0$, and write, for any $0<\varepsilon<\delta$

$$
\begin{aligned}
\mathbf{P}^{*}\left(\bar{L}\left(X_{n}\right) \in K\right) \leqslant & \mathbf{P}^{*}\left(\bar{L}\left(X_{n}\right) \in K,\left\|I_{\alpha}\left(X_{n}\right)-X_{n}\right\| \leqslant \delta\right) \\
& +\mathbf{P}^{*}\left(\left\|I_{\alpha}\left(X_{n}\right)-X_{n}\right\|>\delta\right) \\
\leqslant & \mathbf{P}^{*}\left(\bar{L}\left(I_{\alpha}\left(X_{n}\right)\right) \in \overline{K^{\delta}}\right) \\
& +\mathbf{P}^{*}\left(\left\|I_{\alpha}\left(X_{n}\right)-X_{n}\right\|>\varepsilon\right) .
\end{aligned}
$$

Here we wrote $\overline{K^{\delta}}$ for the closure of $K^{\delta}$, which is compact (recall that $\bar{L}(E)$ is finite dimensional). That compactness ensures, from straightforward analysis, that

$$
\lim _{\alpha \in \Upsilon} J_{\alpha, \bar{L}}\left(\overline{K^{\delta}}\right)=J_{\bar{L}}\left(\overline{K^{\delta}}\right),
$$

from where there exists $\alpha_{0} \in \Upsilon$ fulfilling for each $\alpha \succeq \alpha_{0}$ :

$$
J_{\alpha, \bar{L}}\left(\overline{K^{\delta}}\right)>J_{\bar{L}}\left(\overline{K^{\delta}}\right)-\delta .
$$

Now choose $\varepsilon>0$ so as $1 / \varepsilon>J\left(\overline{K^{\delta}}\right)$ and $\alpha_{1} \succeq \alpha_{0} \in \Upsilon$ fulfilling (B2). We get from (3.14) and (3.15):

$$
\limsup _{n \rightarrow \infty} \frac{1}{v_{n}} \ln \mathbf{P}^{*}\left(\bar{L}\left(X_{n}\right) \in K\right) \leqslant-J_{\bar{L}}\left(\overline{K^{\delta}}\right)+\delta .
$$


Since $J_{\bar{L}}\left(\overline{K^{\delta}}\right) \rightarrow J_{\bar{L}}(K)$ as $\delta \rightarrow 0$ (by simple topological arguments), assertion (3.13) follows. This proves the closed sets part of Lemma 3.1, and hence concludes the proof of that lemma.

Our next lemma shows that point (D2) of Theorem 3.1 is true under assumptions (B1) and (B2).

Lemma 3.2. Under assumptions (B1) and (B2), for each $\varepsilon>0$, there exists a finite partition $\mathscr{A}$ of $B\left(E^{\prime}\right)$ such that

$$
\limsup _{n \rightarrow \infty} \max _{\mathscr{L} \in \mathscr{A}} \frac{1}{v_{n}} \ln \mathbf{P}^{*}\left(\sup _{L, L^{\prime} \in \mathscr{L}}\left|L^{\prime}\left(X_{n}\right)-L\left(X_{n}\right)\right|>\varepsilon\right) \leqslant-\frac{1}{\varepsilon} .
$$

P r o of. Fix $\varepsilon>0$ and choose by (A2) some $\alpha \in \Upsilon$ fulfilling, for all large $n$,

$$
\mathbf{P}^{*}\left(\left\|I_{\alpha}\left(X_{n}\right)-X_{n}\right\|>\frac{\varepsilon}{4}\right) \leqslant \exp \left(-\frac{v_{n}}{\varepsilon}\right) .
$$

Since $I_{\alpha}(E)$ is a Polish space and by $(\mathrm{A} 1)$, the sequence $\left(I_{\alpha}\left(X_{n}\right)\right)_{n \geqslant 1}$ is LDtight in the sense of $[14$, Lemma 2.6], from where there exists a compact $K$ of $(E,\|\cdot\|)$ fulfilling, for all large $n$,

$$
\mathbf{P}^{*}\left(I_{\alpha}\left(X_{n}\right) \notin K\right) \leqslant \exp \left(-\frac{v_{n}}{\varepsilon}\right) .
$$

But $B\left(E^{\prime}\right)_{\mid K}:=\left\{L_{\mid K}, L \in B\left(E^{\prime}\right)\right\}$ is a set of equicontinuous and uniformly bounded elements of $\left(L^{\infty}(K),\|\cdot\|_{K}\right)$ and hence totally bounded in that space (here, $L_{\mid K}$ denotes the restriction of $L$ to the set $K$ ). This implies that we can construct a finite partition $\mathscr{A}$ of $B\left(E^{\prime}\right)$ such that

$$
\max _{\mathscr{L} \in \mathscr{A}} \sup _{L, L^{\prime} \in \mathscr{L}} \sup _{x \in K}\left|L(x)-L^{\prime}(x)\right|<\frac{\varepsilon}{4} .
$$

Now, combining (3.17), (3.18), and (3.19) we get, for all large $n$ and for all $\mathscr{L} \in \mathscr{A}:$

$$
\begin{aligned}
& \mathbf{P}^{*}\left(\sup _{L, L^{\prime} \in \mathscr{L}}\left|L^{\prime}\left(X_{n}\right)-L\left(X_{n}\right)\right|>\varepsilon\right) \\
& \leqslant \mathbf{P}^{*}\left(\left\|I_{\alpha}\left(X_{n}\right)-X_{n}\right\|>\frac{\varepsilon}{4}\right) \\
& \quad+\mathbf{P}^{*}\left(\sup _{L, L^{\prime} \in \mathscr{L}}\left|L^{\prime}\left(I_{\alpha}\left(X_{n}\right)\right)-L\left(I_{\alpha}\left(X_{n}\right)\right)\right|>\frac{\varepsilon}{4}\right) \\
& \leqslant \mathbf{P}^{*}\left(\left\|I_{\alpha}\left(X_{n}\right)-X_{n}\right\|>\frac{\varepsilon}{4}\right)+\mathbf{P}^{*}\left(I_{\alpha}\left(X_{n}\right) \notin K\right) \\
& \leqslant 2 \exp \left(-\frac{v_{n}}{\varepsilon}\right)
\end{aligned}
$$

which concludes the proof of Lemma 3.2. 
Now combining Lemmas 3.1 and 3.2, we conclude by Theorem 3.1 that $\left(X_{n}\right)_{n \geqslant 1}$ satisfies the LDP in $(E,\|\cdot\|)$ for $v_{n}$ and for the following rate function:

$$
\begin{aligned}
& J(x):=\sup \left\{J_{\bar{L}}(L(x)), p \geqslant 1, L \in B\left(E^{\prime}\right)^{p}\right\} \\
& =\sup \left\{J_{\alpha, \bar{L}}\left(L_{1}(x), \ldots L_{1}(x)\right), \bar{L}=\left(L_{1}, \ldots, L_{p}\right), L_{i} \in B\left(E^{\prime}\right), p \geqslant 1, \alpha \in \Upsilon\right\} \\
& =\sup _{\alpha \in \Upsilon} \sup \left\{J_{\alpha, \bar{L}}\left(L_{1}(x), \ldots L_{1}(x)\right), \bar{L}=\left(L_{1}, \ldots, L_{p}\right), L_{i} \in B\left(E^{\prime}\right), p \geqslant 1\right\} \\
& =\sup _{\alpha \in \Upsilon} J_{\alpha}(x),
\end{aligned}
$$

where the last equality in (3.20) is a consequence of (3.10). This concludes the proof of the first part of Theorem 1.2, namely (B1) together with (B2) imply (b1).

3.2.2. Proof that (b1) implies (B1) and (B2). First note that $(\mathrm{b} 1) \Rightarrow(\mathrm{B} 1)$ is a consequence of the contraction principle. To prove (B2) we shall make use of points 2 and 3 of Theorem 3.1, with the choice of $\lambda:=\sup \left\{\||| I_{\alpha}|| \mid\right\}$. Showing (B2) boils down to showing that

$$
\lim _{\alpha \in \Upsilon} \sup _{L \in B\left(E^{\prime}\right)} \rho_{0}\left(L, L \circ I_{\alpha}\right)=0,
$$

which is equivalent to

$$
\lim _{\alpha \in \Upsilon} \sup _{L \in B\left(E^{\prime}\right)} \rho_{k}\left(L, L \circ I_{\alpha}\right)=0 \quad \text { for each } k \geqslant 1 .
$$

Writing $\Gamma_{k}:=\{e \in E, J(e) \leqslant k\}$ we have, for all $\alpha \in \Upsilon$,

$$
\begin{aligned}
\sup _{L \in B\left(E^{\prime}\right)} \rho_{k}\left(L, L \circ I_{\alpha}\right) & =\sup _{L \in B\left(E^{\prime}\right)} \sup _{e \in \Gamma_{k}}\left|L(e)-L\left(I_{\alpha}(e)\right)\right| \\
& =\sup _{e \in \Gamma_{k}}\left\|e-I_{\alpha}(e)\right\| .
\end{aligned}
$$

Since $\Gamma_{k}$ is a compact subset of $(E,\|\cdot\|)$, which satisfies the BAP with $\left(I_{\alpha}\right)_{\alpha \in \Upsilon}$, assertion (3.21) follows. This concludes the proof of Theorem 1.2.

3.3. Proof of Theorem 2.1. The proof is achieved throughout several lemmas. The first lemma is dedicated to the finite dimensional LDP for $\left(2 \log _{2} n\right)^{-1 / 2} \alpha_{n, K, h_{n}}$.

Lemma 3.3. Assume that the conditions of Theorem 2.1 hold. Then, for any finite collection $0 \leqslant t_{1}<\cdots<t_{p} \leqslant 1$, the sequence

$$
\left\{\left(2 \log _{2} n\right)^{-1 / 2}\left(\alpha_{n, K, h_{n}}\left(t_{1}\right), \ldots, \alpha_{n, K, h_{n}}\left(t_{p}\right)\right)\right\}_{n \geqslant 1}
$$

satisfies the LDP in $\mathbf{R}^{p}$ for $v_{n}=\log _{2} n$ and

$$
J_{t_{1}, \ldots, t_{p}}:\left(x_{1}, \ldots, x_{p}\right) \mapsto \sum_{i=1}^{p-1}\left(t_{i+1}-t_{i}\right)\left(\frac{x_{i+1}-x_{i}}{t_{i+1}-t_{i}}\right)^{2} .
$$


$\mathrm{Pr}$ o of of $\mathrm{L} \mathrm{e} \mathrm{m} \mathrm{m}$ a 3.3. The proof is a straightforward use of the Gärtner-Ellis theorem. We omit details.

The following an exponential inequality for the Hölder modulus of oscillation of a certain class of processes. It is established essentially to check condition (3.26) of Proposition 3.1 (see in what follow), but presents an interest in itself.

Lemma 3.4. Let $X$ be a separable stochastic process satisfying, for some constants $\alpha \in(0,1), \beta \in(0,1), C>0, A>0$, and $v>0$,

$$
\forall t, t^{\prime} \in[0,1], t \neq t^{\prime}, \quad \mathbf{P}\left(\frac{\left|X(t)-X\left(t^{\prime}\right)\right|}{\left|t-t^{\prime}\right|^{\alpha}} \geqslant A\right) \leqslant C \exp \left(\frac{-A^{2} v}{\left|t-t^{\prime}\right|^{\beta}}\right) .
$$

Then for each integer $m$ satisfying $m\left(\ln 2+A^{2} v\right) \leqslant 2^{m \beta} A^{2} v$ we have

$$
\mathbf{P}\left(\sup _{\substack{t, t^{\prime} \in[0,1] \\ 0<\left|t-t^{\prime}\right|<2^{-m}}} \frac{\left|X(t)-X\left(t^{\prime}\right)\right|}{\left|t-t^{\prime}\right|^{\alpha}} \geqslant \frac{A}{1-2^{-\alpha}}\right) \leqslant \frac{C \exp \left(-v A^{2} m\right)}{1-\exp \left(-v A^{2}\right)} .
$$

Pr o of of $\mathrm{L}$ e $\mathrm{mm}$ a 3.4. Consider the events

$$
B_{m}:=\left\{\sup _{m^{\prime} \geqslant m} \max _{0 \leqslant k \leqslant 2^{m^{\prime}}-1} \frac{\left|X\left(t_{k+1, m^{\prime}}\right)-X\left(t_{k, m^{\prime}}\right)\right|}{2^{-m^{\prime} \alpha}} \geqslant A\right\},
$$

where $t_{k, m^{\prime}}:=2^{-m^{\prime}} k$. Bonferroni's inequality together with $m^{\prime}\left(\ln 2+A^{2} v\right) \leqslant$ $2^{m^{\prime} \beta} A^{2} v$ for each $m^{\prime} \geqslant m$ yield

$$
\begin{aligned}
\mathbf{P}\left(B_{m}\right) & \leqslant \sum_{m^{\prime}=m}^{\infty} C \cdot 2^{m^{\prime}} \exp \left(-\frac{v A^{2}}{2^{-m^{\prime} \beta}}\right)=C \sum_{m^{\prime}=m}^{\infty} \exp \left(-\frac{v A^{2}}{2^{-m^{\prime} \beta}}+m^{\prime} \ln 2\right) \\
& \leqslant C \sum_{m^{\prime}=m}^{\infty} \exp \left(-v A^{2} m^{\prime}\right) .
\end{aligned}
$$

Now, for any pair of distinct dyadic points $t, t^{\prime}$ satisfying $t^{\prime}-t<2^{-m}$ we can write $t-t^{\prime}=\sum_{i=1}^{N} 2^{-i+m} \delta_{i}$, where $N$ is integer and $\delta_{i} \in\{0,1\}$. Writing $\Delta_{k}:=\sum_{i=1}^{k} 2^{-i+m} \delta_{i}, k=0, \ldots, N$, we can decompose, on the event $B_{m}^{C}$ :

$$
\begin{aligned}
\left|X\left(t^{\prime}\right)-X(t)\right| & \leqslant \sum_{k=1}^{N}\left|X\left(t+\Delta_{k}\right)-X\left(t+\Delta_{k-1}\right)\right| \leqslant A \sum_{k=1}^{N} \delta_{k} \cdot 2^{-(k+m) \alpha} \\
& \leqslant \frac{A}{1-2^{-\alpha}}\left(\sum_{k=1}^{N} \delta_{k} \cdot 2^{-k+m}\right)^{\alpha}=\frac{A}{1-2^{-\alpha}}\left|t^{\prime}-t\right|^{\alpha} .
\end{aligned}
$$

Since $X$ is separable and the set of dyadic points is dense in $[0,1]$, the proof is concluded.

The following proposition shows that LD-thightness in $H_{0, \alpha}$ can be split in two parts. 
Proposition 3.1. Let $\beta \in(0,1)$ and let $\left(X_{n}\right)_{n \geqslant 1}$ be a sequence of separable stochastic processes having trajectories in $H_{0, \alpha}$ and satisfying, for some sequences of nonrandom positive numbers $a_{n} \downarrow 0$ and $v_{n} \rightarrow \infty$ :

1) $\mathscr{D}\left(X_{n}, \alpha, a_{n}\right)$ is LD-tight for $v_{n}$, namely,

$$
\lim _{M \rightarrow \infty} \limsup _{n \rightarrow \infty} \frac{1}{v_{n}} \ln \mathbf{P}\left(\mathscr{D}\left(X_{n}, \alpha, a_{n}\right) \geqslant M\right)=-\infty ;
$$

2) for each $A>0$, there exists $n_{0}$ such that for any $n \geqslant n_{0}, t, t^{\prime} \in[0,1]$, $\left|t-t^{\prime}\right| \geqslant a_{n}$

$$
\mathbf{P}\left(\frac{\left|X_{n}\left(t^{\prime}\right)-X_{n}(t)\right|}{\left|t^{\prime}-t\right|^{\alpha}} \geqslant A\right) \leqslant \exp \left(-\frac{A^{2} v_{n}}{\left|t^{\prime}-t\right|^{\beta}}\right) .
$$

Then for each $\varepsilon>0$ we have

$$
\lim _{\delta \rightarrow 0} \limsup _{n \rightarrow \infty} \frac{1}{v_{n}} \ln \mathbf{P}\left(\mathscr{D}\left(X_{n}, \alpha, \delta\right) \geqslant \varepsilon\right) \leqslant-\frac{1}{\varepsilon} .
$$

Proof of Proposition 3.1. For fixed $n$, let $\widetilde{X}_{n}$ be the linear interpolation of $X_{n}$ along the grid $t_{k, n}:=k a_{n}, k=0, \ldots,\left[1 / a_{n}\right]$, $t_{\left[1 / a_{n}\right]+1}:=1$. As shown in [11, last lines of p. 395], we have $\mathscr{D}\left(X_{n}-\right.$ $\left.\tilde{X}_{n}, \alpha, 1\right) \leqslant 4 \mathscr{D}\left(X_{n}, \alpha, a_{n}\right)$ almost surely, and hence we just need to show that (3.27) holds with $\widetilde{X}_{n}$ instead of $X_{n}$. To show this we fix $\varepsilon>0$ and take $A=\varepsilon\left(1-2^{-\alpha}\right)$. We shall make use of Lemma 3.4 for $n$ large enough. To verify conditions of Lemma 3.4, take $t \neq t^{\prime}$ with $\left|t^{\prime}-t\right|>a_{n}$ (the case where $\left|t^{\prime}-t\right| \geqslant a_{n}$ is already covered by (3.26)). There are two cases that will be treated separately. The first case is when $t, t^{\prime}$ both belong to a common interval $\left[t_{k, n}, t_{k, n+1}\right]$. By definition of $\widetilde{X}_{n}$ we have

$$
\begin{aligned}
& \mathbf{P}\left(\frac{\left|\widetilde{X}_{n}\left(t^{\prime}\right)-\tilde{X}_{n}(t)\right|}{\left|t^{\prime}-t\right|^{\alpha}} \geqslant A\right) \\
& \quad=\mathbf{P}\left(\frac{\left|X_{n}\left(t_{k+1, n}\right)-X_{n}\left(t_{k, n}\right)\right|}{\left|t_{k+1, n}-t_{k, n}\right|^{\alpha}}\left|\frac{t^{\prime}-t}{t_{k+1, n}-t_{k, n}}\right|^{1-\alpha} \geqslant A\right) \\
& \quad \leqslant \exp \left(-\frac{A^{2}\left|t_{k+1, n}-t_{k, n}\right|^{2-2 \alpha} v_{n}}{\left|t^{\prime}-t\right|^{2-2 \alpha}\left|t_{k+1, n}-t_{k, n}\right|^{\beta}}\right) \\
& \quad=\exp \left(-\frac{A^{2} v_{n}}{\left|t^{\prime}-t\right|^{\beta}}\left|\frac{t_{k+1, n}-t_{k, n}}{t^{\prime}-t}\right|^{2-2 \alpha-\beta}\right) \\
& \quad \leqslant \exp \left(-\frac{A^{2} v_{n}}{\left|t^{\prime}-t\right|^{\beta}}\right),
\end{aligned}
$$

where the last equality in (3.28) holds since $\left|t^{\prime}-t\right| \leqslant\left|t_{k+1, n}-t_{k, n}\right|$ and $2-2 \alpha-\beta \geqslant 0$. The second case to be treated is when there exists exactly one $t_{k, n}$ fulfilling $t \leqslant t_{k, n}<t^{\prime}$. In that case

$$
\frac{\left|\tilde{X}_{n}\left(t^{\prime}\right)-\tilde{X}_{n}(t)\right|}{\left|t^{\prime}-t\right|^{\alpha}} \leqslant \frac{\left|\widetilde{X}_{n}\left(t^{\prime}\right)-\tilde{X}_{n}\left(t_{k, n}\right)\right|}{\left|t^{\prime}-t_{k, n}\right|^{\alpha}}+\frac{\left|\tilde{X}_{n}\left(t_{k, n}\right)-\widetilde{X}_{n}(t)\right|}{\left|t_{k, n}-t\right|^{\alpha}},
$$


which proves that $\tilde{X}_{n}$ satisfies the conditions of Lemma 3.4 (with $C=2$ and $\left.v=v_{n} / 4\right)$, by making use of (3.28) together with the triangle inequality for probabilities. The proof is concluded by choosing $m$ large enough to have $\varepsilon^{2}\left(1-2^{-\alpha}\right)^{2} m / 4>1 / \varepsilon$.

The two following lemmas are devoted to show that $\left(2 \log _{2} n\right)^{-1 / 2} \alpha_{n, K, h_{n}}$ satisfies conditions (3.25) and (3.26) of Proposition 3.1.

Lemma 3.5. For any sequences of real numbers $a_{n}$ and $h_{n}$ vanishing at $\infty$, and fulfilling $a_{n}^{(1-\alpha) / 2}=O\left(h_{n}\right)$ the sequence $\mathscr{D}\left(\alpha_{n, K, h_{n}} / \sqrt{2 \log _{2} n}, \alpha, a_{n}\right)$ is LD-tight for $v_{n}=\log _{2} n$.

$\mathrm{P}$ r o o f of $\mathrm{L}$ e $\mathrm{m} \mathrm{m}$ a 3.5. Recalling that $K$ is Lipschitz we have, for any $t, t^{\prime} \in[0,1]$,

$$
\begin{aligned}
\left|\alpha_{n, K, h_{n}}\left(t^{\prime}\right)-\alpha_{n, K, h_{n}}(t)\right| & =\int_{[0,1]}\left|\alpha_{n}(s)\right|\left|K_{h}\left(t^{\prime}-s\right)-K_{h}(t-s)\right| d s \\
& \leqslant \int_{[0,1]}\left|\alpha_{n}(s)\right| d s \times C^{\prime} \frac{\left|t^{\prime}-t\right|}{h_{n}^{2}},
\end{aligned}
$$

from where

$$
\mathscr{D}\left(\frac{\alpha_{n, K, h_{n}}}{\sqrt{2 \log _{2} n}}, \alpha, a_{n}\right) \leqslant \int_{[0,1]} \frac{\left|\alpha_{n}(s)\right|}{\sqrt{2 \log _{2} n}} d s C^{\prime} \frac{a_{n}^{1-\alpha}}{h_{n}^{2}} \leqslant \frac{\left\|\alpha_{n}\right\|_{[0,1]}}{\sqrt{2 \log _{2} n}} C^{\prime} \frac{a_{n}^{1-\alpha}}{h_{n}^{2}},
$$

where $C^{\prime}$ is the Lipschitz constant of $K$.

This last quantity forms a LD-tight sequence for $v_{n}=\log _{2} n$, by a straightforward use of the Dworetzky-Kiefer-Wolfowitz inequality [8]. Lemma 3.5 is proved.

Lemma 3.6. Assume that $a_{n}$ fulfills $\log _{2} n=o\left(n a_{n}^{2(1-\alpha)}\right)$. Then, given $A>0$ we have, for all $n$ large enough,

$$
\begin{gathered}
\forall t, t^{\prime},\left|t^{\prime}-t\right| \geqslant a_{n} \\
\mathbf{P}\left(\frac{\left|\alpha_{n, K, h_{n}}\left(t^{\prime}\right)-\alpha_{n, K, h_{n}}(t)\right|}{\sqrt{2 \log _{2} n}\left|t^{\prime}-t\right|^{\alpha}} \geqslant A\right) \leqslant \exp \left(-\frac{A^{2} \log _{2} n}{4\left|t^{\prime}-t\right|^{1-2 \alpha}}\right) .
\end{gathered}
$$

P r o of of $\mathrm{L}$ e $\mathrm{m} \mathrm{m}$ a 3.6. Write $G(x)=\int_{-x}^{\infty} K(u) d u$ and

$$
\sigma_{n}^{2}\left(t, t^{\prime}\right)=\mathbf{D}\left[G\left(\frac{t^{\prime}-U_{i}}{h_{n}}\right)-G\left(\frac{t-U_{i}}{h_{n}}\right)\right] .
$$

First notice that, for fixed $t, t^{\prime}$, the left-hand side of (3.29) can be written as

$$
\begin{aligned}
& \mathbf{P}\left(\left|\sum_{i=1}^{n}\left(\left[G\left(\frac{t^{\prime}-U_{i}}{h_{n}}\right)-G\left(\frac{t-U_{i}}{h_{n}}\right)\right]-\mathbf{E},\left[G\left(\frac{t^{\prime}-U_{i}}{h_{n}}\right)-G\left(\frac{t-U_{i}}{h_{n}}\right)\right]\right)\right|\right. \\
& \left.\geqslant A\left|t^{\prime}-t\right|^{\alpha} \sqrt{2 n \log _{2} n}\right) \text {. }
\end{aligned}
$$


According to $\left[11\right.$, p. 400] we have $\sigma_{n}^{2}\left(t, t^{\prime}\right) \leqslant\left|t^{\prime}-t\right|$ for each $n \geqslant 0, t, t^{\prime} \in[0,1]$. Now Bennett's inequality yields

$\mathbf{P}\left(\frac{\left|\alpha_{n, K, h_{n}}\left(t^{\prime}\right)-\alpha_{n, K, h_{n}}(t)\right|}{\sqrt{2 \log _{2} n}\left|t^{\prime}-t\right|^{\alpha}} \geqslant A\right) \leqslant \exp \left(-n\left|t^{\prime}-t\right| h\left(\frac{A \sqrt{2 \log _{2} n}\left|t^{\prime}-t\right|^{\alpha}}{\sqrt{n}\left|t^{\prime}-t\right|}\right)\right)$,

with $h(x):=(1+x) \ln (1+x)-x$. But $\left(\log _{2} n\right)^{-1} n\left|t^{\prime}-t\right|^{2(1-\alpha)} \rightarrow \infty$ uniformly in $\left|t^{\prime}-t\right| \geqslant a_{n}$ hence we can apply $h(x) \sim x^{2} / 2$ as $x \rightarrow 0$ and conclude the proof.

End of the proof of Theorem 2.1. We take $a_{n}:=$ $h_{n}^{2 /(1-\alpha)}$, which allows us to apply Lemmas 3.5 and 3.6 which, through Lemma 3.4, show that $\left(2 \log _{2} n\right)^{-1 / 2} \alpha_{n, K, h_{n}}$ fulfills conditions of Proposition 3.1. That proposition together with Lemma 3.3 implies Theorem 2.1 via Corollary 2.1.

\section{REFERENCES}

1. Arcones M. The large deviation principle for stochastic processes. I. - Теория вероятн. и ее примен., 2002, т. 47, в. 4, с. 567-583.

2. Arcones M. The large deviation principle for stochastic processes. II. - Теория вероятн. и ее примен., 2003, т. 48, в. 1, с. 122-150.

3. Берг Й., Лёфстрем Й. Интерполяционные пространства: Введение. М.: Мир, 1980, $264 \mathrm{c}$.

4. Billingsley P. Convergence of probability measures. New York: Wiley, 1968, $253 \mathrm{p}$.

5. Chernoff $H$. A measure of asymptotic efficiency for tests of a hypothesis based on the sum of observations. - Ann. Math. Statist., 1952, v. 23, № 4, p. 493-507.

6. Cramér H. Sur un nouveau théorème limite de la théorie des probabilités. - Actual. Sci. Industr., 1938, v. 736, p. 5-23.

7. Dembo A., Zeitouni O. Large Deviations Techniques and Applications. Boston: Jones and Bartlett, 1993, $346 \mathrm{p}$.

8. Dvoretzky A., Kiefer J., Wolfowitz J. Asymptotic minimax character of the sample distribution function and of the classical multinomial estimator. - Ann. Math. Statist., 1956, v. 27, № 3, p. 642-669.

9. Ellis R. S. Large deviations for a general class of random vectors. - Ann. Probab., 1984, v. 12, № 1, p. 1-12.

10. Finkelstein $H$. The law of the iterated logarithm for empirical distributions. - Ann. Math. Statist., 1971, v. 42, № 2, p. 607-615.

11. D. Hamadouche, Weak convergence of smoothed empirical processes in Hölder spaces. - Statist. Probab. Lett., 1998, v. 36, № 4, p. 393-400.

12. Hoffmann-Jørgensen J. Stochastic Processes on Polish Spaces. Aarhus: Aarhus Universitet, 1991, 278 p. (Various Publications Ser., v. 39.)

13. Lindenstrauss J., Tzafriri L. Classical Banach Spaces. I. Berlin-New York: SpringerVerlag, 1997, 188 p.

14. Lynch J., Sethuraman J. Large deviations for processes with independent increments. - Ann. Probab., 1987, v. 15, № 2, p. 610-627.

15. Пухальский $A . A$. К теории больших уклонений. - Теория вероятн. и ее примен., 1993 , т. 38 , в. 3, с. 553-562.

16. Singer I. Bases in Banach Spaces. II. Berlin-New York: Springer-Verlag, 1981, 880 p.

17. Suquet C. Tightness in Schauder decomposable Banach spaces. - Amer. Math. Soc. Transl., 1999, v. 193, p. 201-224. 
18. Van der Vaart A., Wellner J. Weak Convergence and Empirical Processes. New York: Springer-Verlag, 1996, 508 p.

19. Varron D., Une remarque concernant les principes de grandes déviations dans les espaces Schauder décomposables. - C.R. Math. Acad. Sci. Paris, 2006, v. 343, № 5, p. $345-348$.

Поступила в редакцию

Исправленный вариант

27.VI.2012 